\title{
Estimating the refractive index of oxygenated and deoxygenated hemoglobin using genetic algorithm - support vector regression model
}

\begin{abstract}
Background and objectives: The refractive index of hemoglobin plays important role in hematology due to its strong correlation with the pathophysiology of different diseases. Measurement of the real part of the refractive index remains a challenge due to strong absorption of the hemoglobin especially at relevant high physiological concentrations. So far, only a few studies on direct measurement of refractive index have been reported and there are no firm agreements on the reported values of refractive index of hemoglobin due to measurement artifacts. In addition, it is time consuming, laborious and expensive to perform several experiments to obtain the refractive index of hemoglobin. In this work, we proposed a very rapid and accurate computational intelligent approach using Genetic Algorithm/Support Vector Regression models to estimate the real part of the refractive index for oxygenated and deoxygenated hemoglobin samples. Methods: These models utilized experimental data of wavelengths and hemoglobin concentrations in building highly accurate Genetic Algorithm/Support Vector Regression model (GA-SVR).Results:The developed methodology showed high accuracy as indicated by the low root mean square error values of $4.65 \times 10-4$ and $4.62 \times 10^{-4}$ for oxygenated and deoxygenated hemoglobin, respectively. In addition, the models exhibited 99.85 and $99.84 \%$ correlation coefficients (r) for the oxygenated and deoxygenated hemoglobin, thus, validating the strong agreement between the predicted and the experimental results. Conclusions: Due to the accuracy and relative simplicity of the proposed models, we envisage that these models would serve as important references for future studies on optical properties of blood.
\end{abstract}

Keyword: Refractive index; Hemoglobin; Genetic algorithm; Support Vector Regression 